\title{
Efeitos Paramétricos do Atraso do Reforço Sobre o Responder de Ratos Submetidos a Esquemas de Razão e Intervalos Variáveis
}

\author{
Cristina Moreira Fonseca \\ Gerson Yukio Tomanari \\ Universidade de São Paulo
}

\begin{abstract}
RESUMO
Foram investigados os efeitos produzidos pela introdução de diferentes valores de atraso do reforço sobre as respostas de pressão à barra emitidas por oito ratos expostos a esquemas de intervalo e razão variáveis (VI e VR, respectivamente). A partir de condições de linha de base sob reforçamento imediato, aplicou-se o atraso do reforço não sinalizado e não reiniciável pelo tempo máximo programado de $1 \mathrm{~s}, 5 \mathrm{~s}$ e $8 \mathrm{~s}$ a cada um dos esquemas, em diferentes condições, em ordens crescente e decrescente de atraso. Os resultados mostraram correlação negativa entre taxas de resposta e valores do atraso do reforço para praticamente todos os sujeitos experimentais, em todos os valores de atraso. Por meio da análise dos atrasos efetivamente obtidos na sessão, foi possível constatar que a distribuição de frequência dos atrasos, independentemente do esquema em vigor, VI ou VR, concentraram-se em $0,5 \mathrm{~s}$, quando o atraso programado era de $2 \mathrm{~s}$. Diferentemente, foram próximos de $5 \mathrm{~s}$ e $8 \mathrm{~s}$ quando os atrasos programados foram de $5 \mathrm{~s}$ e $8 \mathrm{~s}$, respectivamente. Esses efeitos diferenciados do atraso sobre o responder podem ser compreendidos mediante uma análise das alterações produzidas pelo atraso nas contingências específicas de cada esquema.
\end{abstract}

Palavras-chave: atraso do reforço; esquemas de reforço; razão variável; intervalo variável; ratos.

\section{ABSTRACT \\ Parametrical Effects of Delayed Reinforcement on the Responding of Rats Exposed to Variable Interval and Variable Ratio Schedules}

This study examined the effects of introducing different delays of reinforcement on the rate of lever pressing by eight rats under variable interval and variable ratio schedules (VI and VR, respectively). Following baseline conditions under immediate reinforcement, unsignaled and non-resetting delays of reinforcement were introduced upon each of these schedules. In different conditions, delays of $2 \mathrm{~s}, 5 \mathrm{~s}$ and $8 \mathrm{~s}$ were presented in both increasing and decreasing sequences. The results showed negative correlations between rate of responding and delay of reinforcement for most subjects and delays. By analyzing the frequency distribution of the delays actually obtained in each single reinforcer delivery, it was possible to verify a higher concentration of 0.5 -s delays when a 2-s delay was programmed. In contrast, actual delays concentrated around $5 \mathrm{~s}$ and $8 \mathrm{~s}$ when the programmed delays were $5 \mathrm{~s}$ and $8 \mathrm{~s}$, respectively. Such differential effects generated by the delays of reinforcement on responding can be understood by analyzing how the delay affected the ongoing contingencies in each particular schedule.

Keywords: delay of reinforcement; schedules of reinforcement; variable interval; variable ratio; rats.

Em uma contingência operante, o tempo entre a emissão da resposta e a liberação da consequência produz efeitos preponderantes sobre o fortalecimento ou o enfraquecimento desta relação e, portanto, sobre a probabilidade de que a resposta volte a ser emitida (Dews, 1960; Ferster, 1953; Ferster \& Skinner, 1957; Keller \& Schoenfeld, 1950). Estes efeitos têm sido amplamente estudados por meio de procedimentos denominados atraso do reforço, nos quais o tempo entre a resposta e a consequência por ela produzida é manipulado (Dickinson, Watt, \& Griffiths, 1992; Gleeson \& Lattal, 1987; Lattal, 1987; Morgan, 1970; Schneider, 1990; Sizemore \& Lattal, 1977; Williams, 1976).

Para investigar os efeitos do atraso do reforço sobre o responder, utiliza-se basicamente um esquema 
tandem ou encadeado (Catania, 1999). No primeiro elo, respostas que cumpram as exigências do esquema colocam em vigor o segundo elo. No segundo elo, vigora um esquema de tempo fixo ou variável cujo valor determina o atraso do reforço. Para garantir que não ocorram respostas durante o atraso, mantendo-o, portanto, inalterado, aplica-se uma contingência de DRO (Differential reinforcement of other behavior; ver Catania, 1999). Nesse caso, a cada resposta emitida no segundo elo, reinicia-se o atraso - fato que levou esse procedimento a ser denominado de atraso reiniciável ou resetting (Azzi, Fix, Keller, Rocha \& Silva, 1964; Lattal \& Ziegler, 1982).

De especial relevância ao presente trabalho, os efeitos do atraso do reforço são também estudados por outro procedimento, o de atraso não reiniciável ou non-resetting. Neste, diferentemente do procedimento de atraso reiniciável, podem ocorrer respostas durante o período de atraso e estas nunca o reiniciam. Em vista disso, a resposta que efetivamente produz o reforço não é necessariamente a última resposta que o precede e, portanto, o efetivo valor do atraso pode não corresponder exatamente àquele programado pelo experimentador (Schneider, 1990).

Em termos de resultados, estudos experimentais realizados predominantemente com infra-humanos têm mostrado que, em geral, os atrasos do reforço, em ambos procedimentos, são acompanhados por uma diminuição nas taxas de respostas em relação à condição de reforço imediato. De modo geral, quanto maior o atraso, menor tende a ser a taxa de resposta que o acompanha, e vice-versa. (Azzi \& col., 1964; Catania \& Keller, 1981; Richards, 1981; Sizemore \& Lattal, 1977, 1978; Williams, 1976).

Os efeitos marcantes e bastante sistemáticos do atraso do reforço sobre o responder configuram o tempo entre a resposta e sua consequência como uma variável crítica na contingência operante. Esta variável, por sua vez, é modulada por outros fatores, entre os quais, por exemplo, está a presença ou a ausência de estímulos discriminativos que possam acompanhar $o$ atraso. Quanto a esse fator, sabemos que contingências sob atrasos sinalizados tendem a gerar taxas de respostas normalmente mais elevadas do que sob atrasos não sinalizados (Dews, 1960; Ferster, 1953; Richards, 1981; Sizemore \& Lattal, 1978).

Outro fator que modula os efeitos do atraso diz respeito aos esquemas de reforçamento em vigor (Pierce, Handford, \& Zimmerman, 1972; Sizemore \& Lattal, 1978). A esse respeito, Fonseca e Tomanari (2007) analisaram os efeitos da introdução de atrasos não reiniciáveis de $5 \mathrm{~s}$ sobre o responder de ratos em dois esquemas distintos, intervalo variável (VI) e razão variável (VR). Os resultados mostraram que, com a introdução do atraso do reforço, houve uma clara diminuição nas taxas de respostas subsequentemente à condição anterior de reforçamento imediato. No entanto, a diminuição foi mais acentuada para os sujeitos cujo responder encontrava-se em VI, comparativamente aos sujeitos cujo responder encontrava-se em VR.

Em prosseguimento ao trabalho de Fonseca e Tomanari (2007), a presente investigação buscou avançar na análise sistemática das variáveis que afetam o responder de ratos sob condições de atraso do reforço. No presente, introduzimos diferentes valores do atraso com vistas a verificar seus possíveis efeitos paramétricos sobre o responder de ratos em VI e VR.

\section{MÉTODO}

\section{Sujeitos}

Foram sujeitos deste trabalho oito ratos ingênuos, albinos (Ratttus norvergicus), Wistar, machos. No início do experimento, os sujeitos tinham entre 100 e 110 dias de vida. Alojados em gaiolas individuais, eram mantidos a $85 \%$ do peso ad libitum por regime de restrição de acesso à água. Alimento estava constantemente disponível.

\section{Equipamento}

Foram usadas quatro caixas de condicionamento operante modulares, modelo ENV-007, fabricadas por Med Associates Inc. As caixas estavam equipadas com um bebedouro localizado no centro da parede direita. Em repouso, o bebedouro era mantido mergulhado em uma cuba d'água localizada na parte externa da caixa. A cada acionamento do bebedouro, uma concha com cerca de $0,06 \mathrm{ml}$ de água era introduzida no interior da caixa, onde permanecia por $4 \mathrm{~s}$. À direita do bebedouro, encontrava-se uma barra de respostas. Localizada na parede oposta ao bebedouro, havia uma lâmpada que produzia a iluminação ambiente durante a sessão experimental.

As sessões experimentais foram controladas por um microcomputador compatível com IBM-PC, uma interface (DIG-700P1) e o aplicativo Windows MedPC (SOF-735), os quais, a partir de rotinas de programação desenvolvidas no programa Turbo Pascal v. 1.5, em linguagem MedState Notation, comandaram as contingências nas caixas experimentais e registraram as respostas de pressão à barra. 


\section{Procedimento}

Inicialmente, todos os oito sujeitos passaram por um treino inicial que envolveu as seguintes etapas: treino ao bebedouro, modelagem da resposta de pressão à barra, fortalecimento da resposta em esquema de reforçamento contínuo e, por fim, treino do responder em esquema intermitente, nessa ordem. Nesta última etapa, os sujeitos foram distribuídos em dois grupos que se diferenciavam em função do esquema intermitente a que seriam expostos, Grupo VI e Grupo VR. A partir do reforçamento contínuo, os sujeitos do Grupo VI foram submetidos diretamente a VI $20 \mathrm{~s}$. Os sujei- tos do Grupo VR, diferentemente, foram submetidos a razões aumentadas progressivamente ao longo das sessões, isto é, VR 3, VR 6, VR 10 e VR 15.

$\mathrm{Na}$ última etapa do treino inicial, bem como ao longo de todas as fases experimentais, as sessões eram encerradas após a liberação de 100 gotas de água. $\mathrm{O}$ encerramento do treino inicial, bem como mudanças de fases (ver Tabela 1), ocorriam mediante estabilidade na taxa de respostas avaliada por inspeção visual, garantindo-se, nas três últimas sessões, a inexistência de tendências e variações maiores do que $10 \% \mathrm{em}$ relação à média obtida entre as mesmas.

TABELA 1

Distribuição dos Sujeitos nos Grupos VI e VR e Valores do Atraso ao Longo das Fases Experimentais

\begin{tabular}{ccccccc}
\hline \multirow{7}{*}{ Ratos } & \multicolumn{5}{c}{ Fases } \\
\cline { 2 - 6 } & 1 & 2 & 3 & 4 & 5 \\
\hline \multicolumn{7}{c}{ Grupo VI } \\
\hline 157 & VI 20 & tand VI 20 FT 2 & VI 20 & tand VI 20 FT 5 & VI 20 & tand VI 20 FT 8 \\
158 & VI 20 & tand VI 20 FT 2 & VI 20 & tand VI 20 FT 5 & VI 20 & tand VI 20 FT 8 \\
159 & VI 20 & tand VI 20 FT 8 & VI 20 & tand VI 20 FT 5 & VI 20 & tand VI 20 FT 2 \\
160 & VI 20 & tand VI 20 FT 8 & VI 20 & tand VI 20 FT 5 & VI 20 & tand VI 20 FT 2 \\
\hline
\end{tabular}

Grupo VR

\begin{tabular}{lllllll}
\hline 163 & VR 15 & tand VR 15 FT 2 & VR 15 & tand VR 15 FT 5 & VR 15 & tand VR 15 FT 8 \\
164 & VR 15 & tand VR 15 FT 2 & VR 15 & tand VR 15 FT 5 & VR 15 & tand VR 15 FT 8 \\
166 & VR 15 & tand VR 15 FT 8 & VR 15 & tand VR 15 FT 5 & VR 15 & tand VR 15 FT 2 \\
167 & VR 15 & tand VR 15 FT 8 & VR 15 & tand VR 15 FT 5 & VR 15 & tand VR 15 FT 2 \\
\hline
\end{tabular}

\section{Grupo VI}

A partir de fases de linha de base em VI $20 \mathrm{~s}$ (Fases 1, 3 e 5), seguiram-se as fases em tandem VI $20 \mathrm{~s}$ FT 2 s (Fase 2), tandem VI 20 s FT 5 s (Fase 4) e tandem VI 20 s FT 8 s (Fase 6), para os Ratos 157 e 158. A ordem das Fases 2, 4 e 6 foi invertida para os Ratos 159 e 160 . Sob VI 20 s, um reforço era liberado contingentemente à passagem de um intervalo de tempo médio programado de $20 \mathrm{~s}$, seguido pela emissão de uma resposta de pressão à barra. Os valores dos intervalos entre reforços programados para o VI foram: 2, $4,6,8,10,12,14,16,18,20,22,24,26,28,30,32$,
34,36 e 38 s. Cada intervalo repetiu-se por cinco vezes em cada sessão, com exceção do intervalo de $20 \mathrm{~s}$, que se repetiu por dez vezes.

Sob tandem VI 20 s FT t s, assim que completada a exigência do intervalo corrente do esquema de VI $20 \mathrm{~s}$ (programado exatamente como descrito acima), iniciava-se o tempo de $2 \mathrm{~s}, 5 \mathrm{~s}$ ou $8 \mathrm{~s}$ de atraso do reforço a depender da fase em vigor. Ao fim do atraso, uma gota de água era liberada independentemente de qualquer resposta do sujeito. Respostas durante o FT não tinham consequências programadas, porém eram registradas. 


\section{Grupo VR}

A partir da linha de base em VR 15 (Fases 1, 3 e 5), seguiram-se fases em tandem VR 15 FT 2 s (Fase 2), tandem VR 15 FT 5 s (Fase 4) e tandem VR 15 FT 8 s (Fase 6), para os Ratos 163 e 164. A ordem das Fases 2, 4 e 6 foi invertida para os Ratos 166 e 167.

Sob VR 15, uma gota de água era liberada contingentemente à emissão de um número médio programado de 15 respostas de pressão à barra. Os valores das razões programadas para o VR foram: 1, 3, 5, 7, 9, $11,13,15,17,19,21,23,25,27$ e 29 . Cada razão repetiu-se sete vezes por sessão, com exceção das razões $1,5,9,15$ e 27, que repetiram-se seis vezes.

Sob tandem VR 15 FT t s, assim que completada a exigência do número de respostas corrente do VR 15 (programado exatamente como descrito acima), iniciava-se o tempo de $2 \mathrm{~s}, 5 \mathrm{~s}$ ou $8 \mathrm{~s}$ de atraso do reforço, a depender da fase em vigor, ao fim do qual uma gota de água era liberada. Respostas durante o FT não tinham consequências programadas, porém eram registradas.

\section{RESULTADOS E DISCUSSÃO}

A Figura 1 mostra as taxas de respostas de pressão à barra do Grupo VI (Ratos 157, 158, 159 e 160, nos dois gráficos superiores) e do Grupo VR (Ratos 163, 164, 166 e 167, nos dois gráficos inferiores), nas últimas seis sessões de cada uma das fases de linha de base (VI ou VR) e nas seis primeiras e seis últimas sessões sob atraso do reforço (tand VI FT e tand VR FT). Cada gráfico agrupa curvas de dois sujeitos que passaram por condições experimentais semelhantes. Para cada dupla de sujeito, uma sequência diferente de fases foi seguida.

Nas três fases de linha de base, as taxas de respostas, para todos os sujeitos, mostraram-se normalmente mais elevadas do que nas fases com atraso, com exceção do Rato 167. Com a introdução dos diferentes valores de atraso do reforço $(2 \mathrm{~s}, 5 \mathrm{~s}$ e $8 \mathrm{~s})$, as taxas de respostas, para todos os sujeitos, diminuíram nas primeiras seis sessões e, nas últimas seis, estabilizaram-se em valores normalmente mais baixos do que em linha de base. A exceção foi o Rato 167 do Grupo VR que, sob atraso de $2 \mathrm{~s}$, apresentou taxas de resposta maiores do que na linha de base imediatamente anterior. Para todos os sujeitos do Grupo VI, e para dois sujeitos do Grupo VR (Ratos 163 e 167), a diminuição nas taxas de respostas foi tão mais acentuada quanto maior o valor do atraso, ou seja, com atraso de $8 \mathrm{~s}$, as taxas de respostas foram menores do que com atrasos de $5 \mathrm{~s}$ e $2 \mathrm{~s}$. Por sua vez, com atraso de $5 \mathrm{~s}$, as taxas foram menores do que com atraso de $2 \mathrm{~s}$. Pelo que os dados indicam, portanto, as taxas de respostas de todos os sujeitos variaram parametricamente em relação aos valores dos atrasos e de modo independente quanto a ordem em que eram apresentados nas diferentes fases experimentais. Excepcionalmente, os dados dos Ratos 164 e 166 mostraram taxas menores de resposta sob atraso de $5 \mathrm{~s}$ do que de $8 \mathrm{~s}$. Cada um desses ratos foi exposto a uma sequência distinta de apresentação dos atrasos, isto é, crescente para o Rato 164 e decrescente para o Rato 166, o que enfraquece a possibilidade de que a sequência de fases tenha sido preponderante para esses resultados.

As Figuras $2 \mathrm{a}$ e $2 \mathrm{~b}$ sumariam os efeitos paramétricos acima mencionados dos diferentes valores de atraso sobre as taxas de respostas para todos os sujeitos dos Grupos VI (Figura 2a) e VR (Figura 2b). Nessas duas figuras, é possível verificar claramente a redução sistemática na taxa de respostas em função do aumento do atraso, para praticamente todos os sujeitos, independentemente da ordem de exposição às condições experimentais. Comparado ao atraso de $8 \mathrm{~s}$, o atraso de $2 \mathrm{~s}$ foi sempre acompanhado por taxas mais elevadas de respostas. Com relação ao atraso de $5 \mathrm{~s}$, este normalmente gerou taxas de respostas intermediárias entre os atrasos de $2 \mathrm{~s}$ e $8 \mathrm{~s}$, com exceção aos já citados Ratos 164 e 166.

O emprego do procedimento de atrasos não reiniciáveis faz com que os intervalos efetivamente obtidos entre a apresentação do reforço e a última resposta que o preceda sejam variáveis e dependentes do próprio responder do animal (Schneider, 1990). Em outras palavras, o atraso é tomado como uma variável independente, cujos valores efetivamente obtidos se dão na exposição às contingências em vigor. Para lidar com o fato de o atraso efetivamente registrado ser diferente do atraso máximo programado, é preciso utilizar recursos metodológicos que permitam acompanhar os efeitos de uma variável independente que assume os seus valores dinamicamente nas próprias sessões experimentais. No caso presente, contamos com equipamentos computadorizados que permitiram o controle das contingências e a precisão da medida dos atrasos efetivamente consolidados a cada reforço.

A representação gráfica dessa medida encontra-se nas Figuras $3 a$ e $3 b$ que mostram a distribuição de frequência dos atrasos do reforço efetivamente registrados segundo a média obtida nas seis últimas sessões sob VI (Figura 3a) e VR (Figuras 3b). Cada conjunto horizontal de gráficos diz respeito a um dos valores máximos programados do atraso, isto é, $2 \mathrm{~s}, 5 \mathrm{~s}$ e $8 \mathrm{~s}$, respectivamente. 


\section{Painel A}
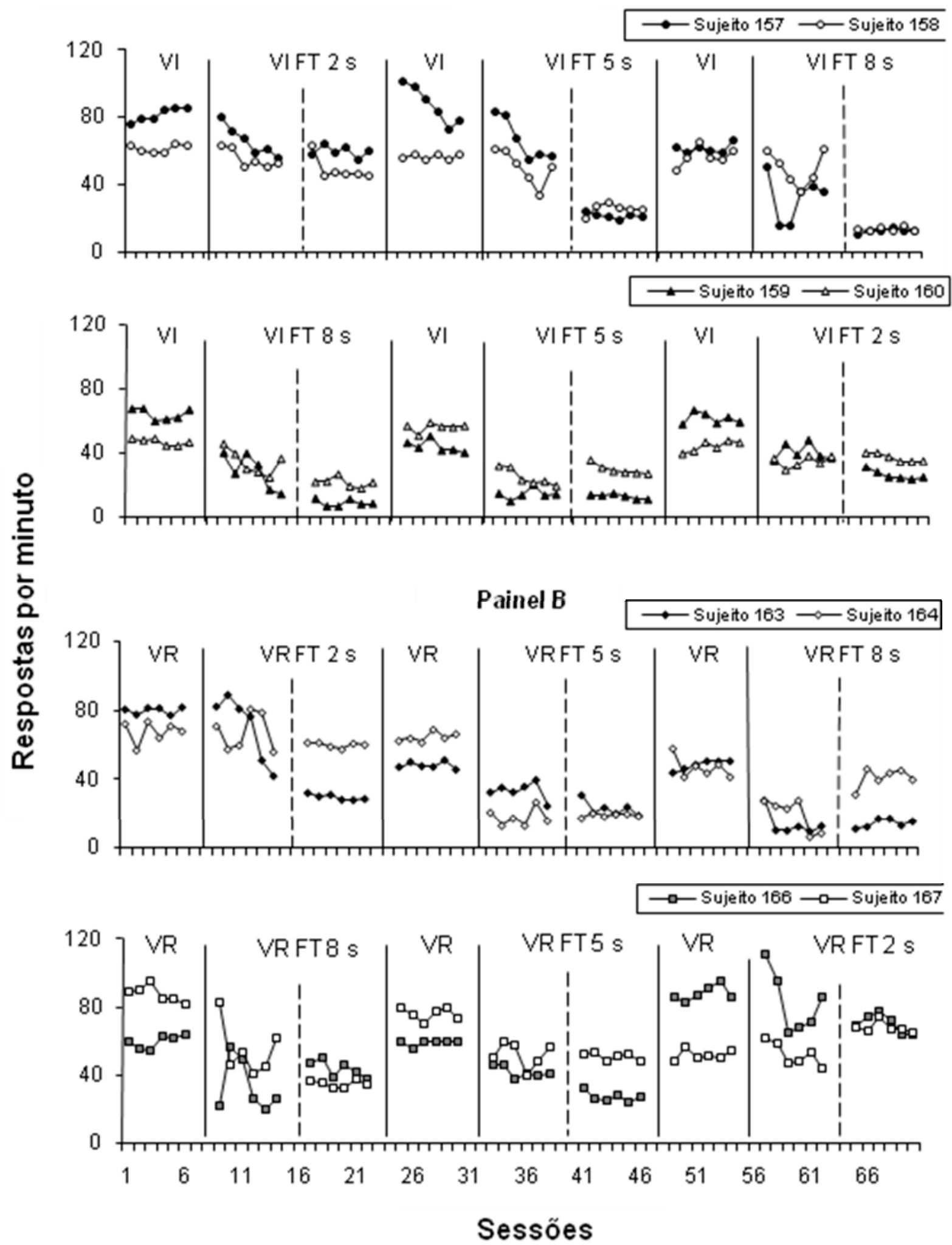

Figura 1. Taxa de resposta de pressão à barra dos sujeitos do Grupo VI (Painel A, gráficos superiores) e do Grupo VR (Painel B, gráficos inferiores) nas seis últimas sessões de cada fase de linha de base e, nas seis primeiras e seis últimas sessões com atrasos $2 \mathrm{~s}, 5 \mathrm{~s}$ e $8 \mathrm{~s}$. Na figura, as linhas sólidas separam as diferentes condições e as linhas tracejadas separam as seis primeiras das seis últimas sessões da mesma fase. 
GRUPO VI

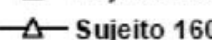

Figura 2a. Taxa média de respostas nas últimas seis sessões com atrasos de $2 \mathrm{~s}, 5 \mathrm{~s}$ e $8 \mathrm{~s}$ para os sujeitos do Grupo Vl.

GRUPO VI

\section{GRUPO VR}

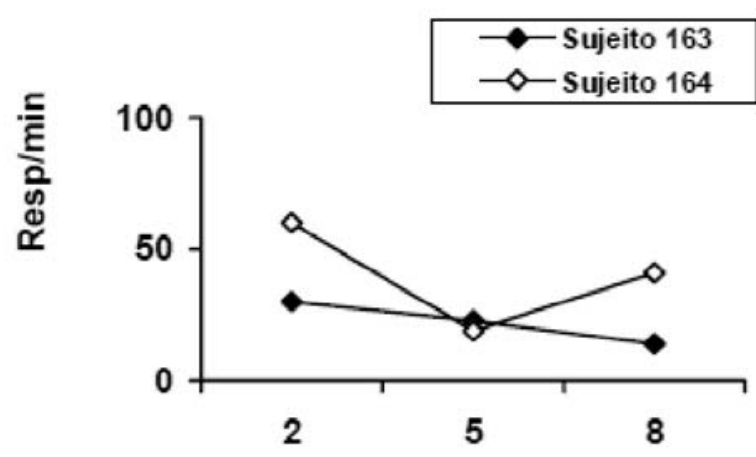

GRUPO VR

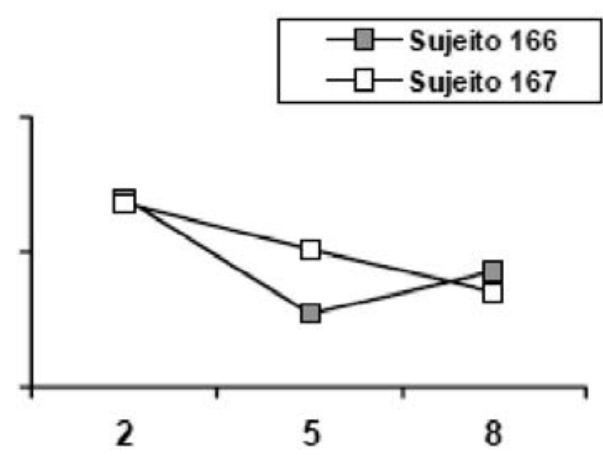

atrasos programados (segundos)

Figura 2b. Taxa média de respostas nas últimas seis sessões com atrasos de $2 \mathrm{~s}, 5 \mathrm{~s}$ e 8 s para os sujeitos do Grupo VR. 


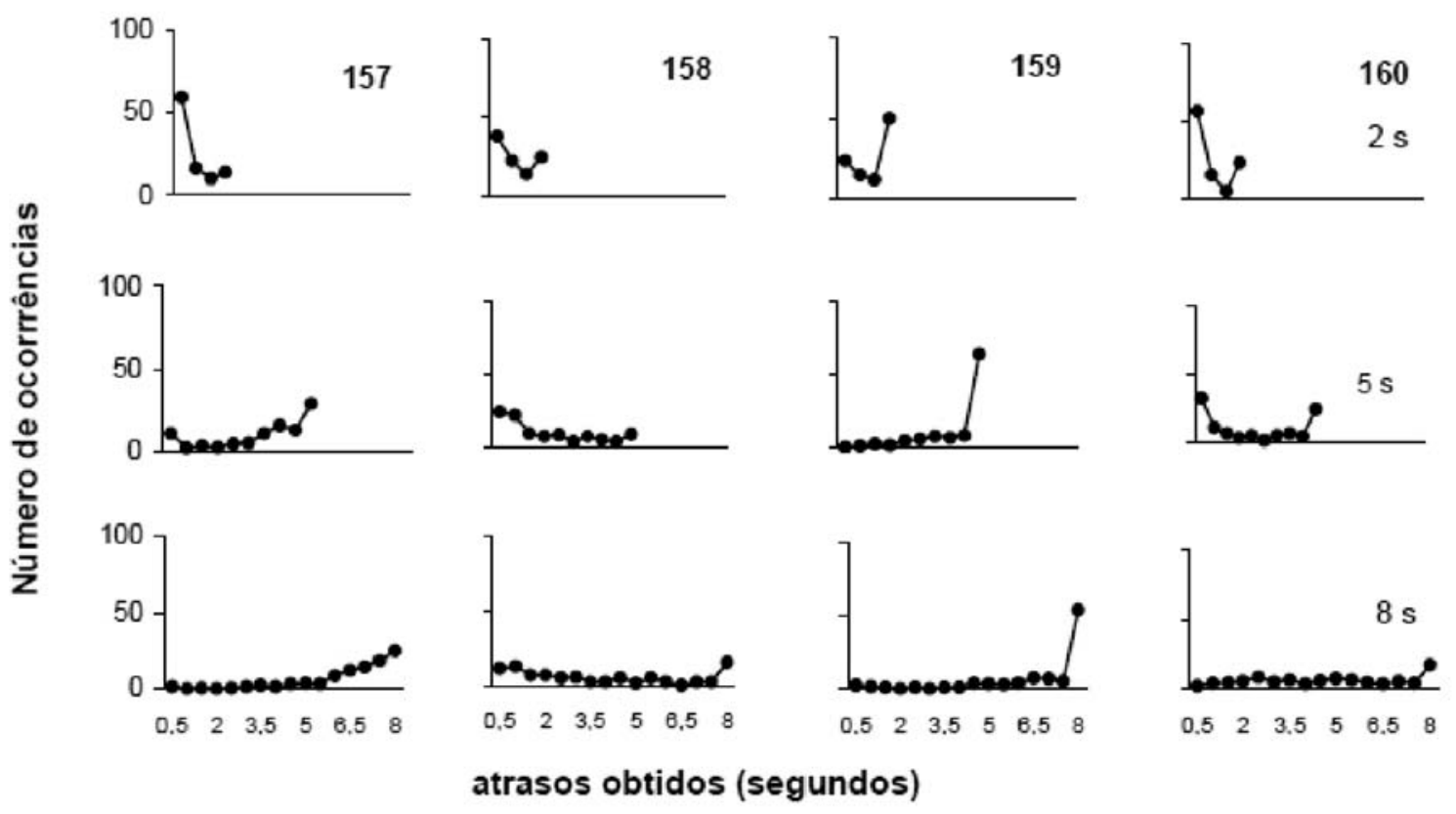

Figura 3a. Distribuição média de respostas em função dos atrasos efetivamente obtidos pelos sujeitos do Grupo VI.
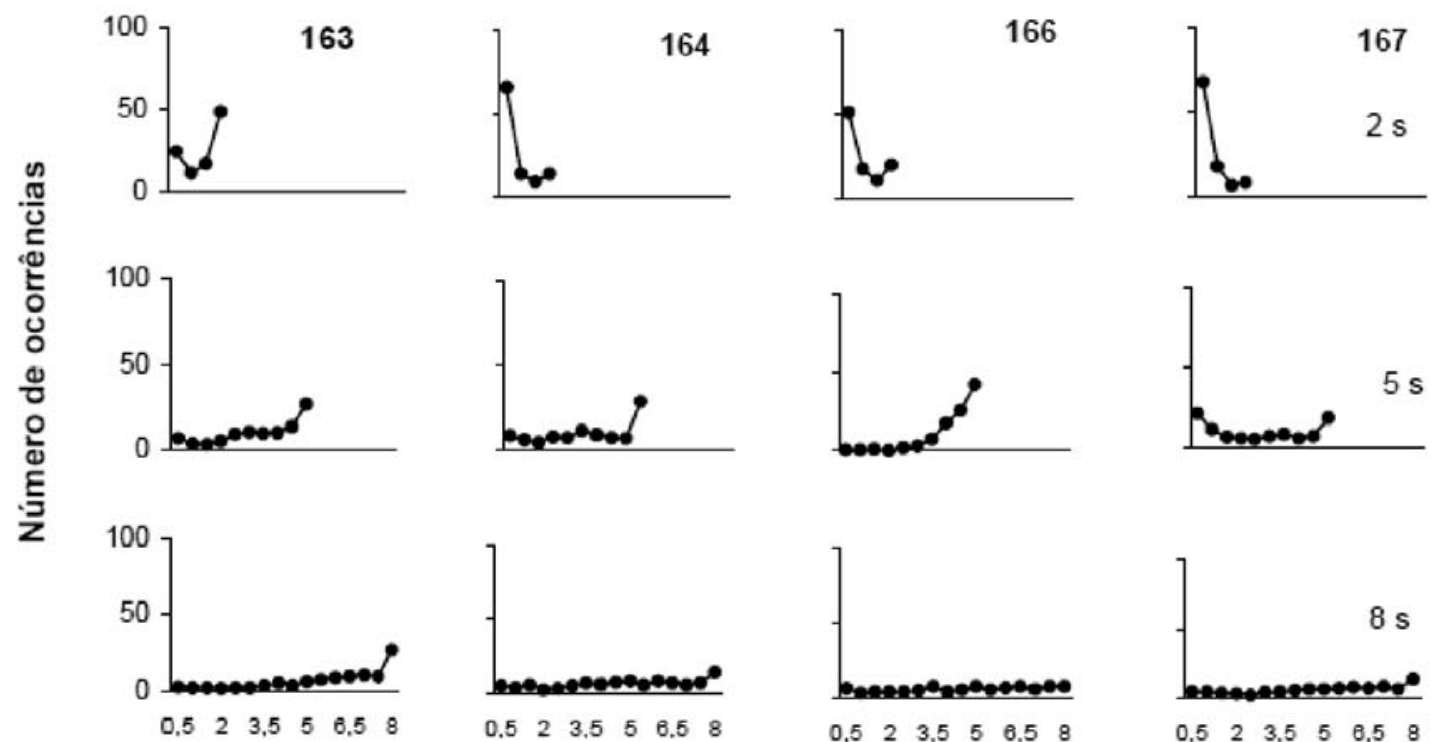

$\begin{array}{lllllll}0,5 & 2 & 3,5 & 5 & 6,5 & 8\end{array}$
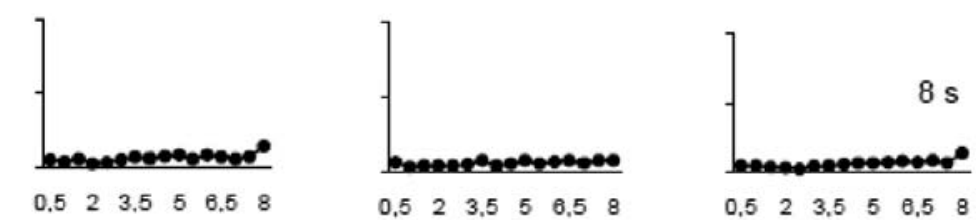

\section{atrasos obtidos (segundos)}

Figura 3b. Distribuição média de respostas em função dos atrasos efetivamente obtidos pelos sujeitos do Grupo VR. 
As distribuições de respostas dos sujeitos dos Grupos VI e VR mostram que, em ambos os grupos, os atrasos efetivamente registrados foram relativamente semelhantes entre si, independentemente do esquema utilizado. De modo geral, os atrasos registrados tenderam a se igualar aos valores programados quando estes últimos eram de $5 \mathrm{~s}$ ou de $8 \mathrm{~s}$. Para atrasos programados de $2 \mathrm{~s}$, diferentemente, os atrasos registrados tenderam a se concentrar em torno de $0,5 \mathrm{~s}$ e a decrescer à medida que o tempo se aproximava de $2 \mathrm{~s}$, tornando os reforços praticamente imediatos à resposta precedente para a maioria dos sujeitos. De modo geral, portanto, ainda que os atrasos tenham sido variáveis, ao contrário do que aconteceria em um procedimento de atraso reiniciável, os valores que assumem na exposição às contingências são compatíveis com as taxas de respostas obtidas. Ou seja, retornando às figuras anteriores, podemos ver que, quanto menor o intervalo efetivamente registrado entre a resposta e o evento consequente (Figuras $3 \mathrm{a}$ e $3 \mathrm{~b}$ ), mais proeminentes tenderam a ser os efeitos do reforçador sobre $o$ responder (Figuras $2 \mathrm{a}$ e $2 \mathrm{~b}$ ).

\section{CONCLUSÕES}

Os dados do presente estudo mostram claramente a correlação inversa entre taxas de respostas e valores do atraso do reforço para praticamente todos os sujeitos experimentais. A introdução de um atraso fixo não reiniciável e não sinalizado de $2 \mathrm{~s}, 5 \mathrm{~s}$ e $8 \mathrm{~s}$ foi acompanhada pela clara redução nas taxas de respostas sob os esquemas de VI 20 s e VR 15. Para o Grupo VI, esse resultado se deu para todos os sujeitos. Para o Grupo VR, esses resultados foram igualmente observados para os Ratos 163 e 167, mas não completamente para os Ratos 164 e 166 (ver discussão adiante). De modo geral, portanto, os resultados replicam dados da literatura no que diz respeito aos efeitos específicos do atraso em diferentes esquemas de reforço (Azzi \& col., 1964; Catania \& Keller, 1981; Fonseca \& Tomanari, 2007; Lattal, 1987; Lattal \& Glesson, 1990; Richards, 1981; Sizemore \& Lattal, 1978, entre outros).

Em particular, a presente investigação utilizou um procedimento de atraso não reiniciável, tornando imprescindível analisar os valores de atraso efetivamente obtidos a cada acionamento do bebedouro. Essa análise permitiu verificar que a distribuição de frequência dos atrasos, independentemente do esquema em vigor, VI ou VR, concentraram-se em $0,5 \mathrm{~s}$ quando o atraso programado era de $2 \mathrm{~s}$. Diferentemente, foram próxi- mos de $5 \mathrm{~s}$ e $8 \mathrm{~s}$ quando os atrasos foram de $5 \mathrm{~s}$ e $8 \mathrm{~s}$, respectivamente. Para compreendermos esses efeitos diferenciados dos atrasos sobre o responder, é preciso analisar as alterações que os mesmos produziram nas diferentes contingências em vigor.

Introduzido o atraso no VI 20 s, parâmetros temporais desse esquema foram inevitavelmente alterados (Lattal, 1987; Morgan, 1970). Quando em vigor os atrasos de $2 \mathrm{~s}, 5 \mathrm{~s} \mathrm{e} 8 \mathrm{~s}$, o menor intervalo entre reforços passou de $2 \mathrm{~s}$ para $4 \mathrm{~s}, 7 \mathrm{~s}$ e $10 \mathrm{~s}$, respectivamente. Por sua vez, o maior intervalo entre reforços passou de $38 \mathrm{~s}$ para $40 \mathrm{~s}, 43 \mathrm{~s}$ e $46 \mathrm{~s}$, respectivamente. Além disso, considerando-se que o tempo programado para a liberação de cada um dos reforços aumentou em $2 \mathrm{~s}$, $5 \mathrm{~s}$ e $8 \mathrm{~s}$, a vigência do atraso implicou um aumento no tempo total da sessão em até 3 minutos, 8 minutos e 13 minutos, quando o atraso programado era de $2 \mathrm{~s}$, $5 \mathrm{~s}$ ou $8 \mathrm{~s}$, respectivamente. Em havendo mais tempo, portanto, as oportunidades para emissão de respostas igualmente aumentaram em relação à linha de base.

As oportunidades de resposta propiciadas pelo atraso do reforço foram especialmente relevantes no caso do VR. Nesse esquema de razão, acabou-se por adicionar um requisito temporal que era definido tão somente pelo número de respostas na história do sujeito em linha de base. Assim, considerando que as respostas durante o atraso não o modificavam, tampouco podiam estar sob controle discriminativo, a introdução do atraso resultou em um aumento variável no número de respostas, ainda que este aumento não fizesse parte da razão exigida. Ou seja, relativamente às respectivas linhas de base, o atraso do reforço gerou no VR, mas não no VI, a introdução de um aspecto temporal antes inexistente no esquema, condição em que a obtenção do reforço passou a se dar com um número maior de respostas.

Identificados o que parecem ser os principais efeitos específicos do atraso do reforço sobre as contingências de VI e VR, percebe-se que os mesmos adquirem maior relevância sobre o responder quanto maior o valor do atraso. Por exemplo, em relação ao reforço imediato, o atraso de $8 \mathrm{~s}$, comparativamente ao atraso de 2 s, é acompanhado por um conjunto de distinções: salienta-se o papel temporal acoplado ao esquema de razão; aumenta-se a oportunidade de respostas; aumenta-se a amplitude de variação no número de respostas possivelmente emitidas e, criticamente, aumenta-se a amplitude dos valores de atraso efetivamente registrados. A partir da análise desses fatores, portanto, torna-se possível compreender os resultados 
do presente estudo, no que este mostrou os efeitos paramétricos do atraso do reforço em um mesmo esquema; os efeitos distintos do atraso do reforço em VI e VR anteriormente destacados por Fonseca e Tomanari (2007); os efeitos dos diferentes valores de atraso, em particular no caso do atraso de $2 \mathrm{~s}$ em relação aos atrasos de $5 \mathrm{~s}$ e $8 \mathrm{~s}$.

\section{REFERÊNCIAS}

Azzi, R., Fix, D. S. R., Keller, F. S., \& Rocha e Silva, M. I. (1964). Exteroceptive control of response under delayed reinforcement. Journal of the Experimental Analysis of Behavior, 7, 159-162.

Catania, A. C. (1999). Aprendizagem: Comportamento, linguagem e cognição (D. G. Souza, Trad.). Porto Alegre: Artes Médicas.

Catania, A. C., \& Keller, K. J. (1981). Contingency, contiguity, correlation, and the concept of causation. Em P. Harzem, \& M. D. Zeiler (Orgs.), Predictability, correlation, and contiguity (pp. 125-167). London: Wiley.

Dews, P. B. (1960). Free-operant behavior under conditions of delayed reinforcement. I. CRF-type schedules. Journal of the Experimental Analysis of Behavior, 3, 221-234.

Dickinson, A., Watt, A., \& Griffiths, W. J. H. (1992). Freeoperant acquisition with delayed reinforcement. The Quarterly Journal of Experimental Psychology, 45B, 241-258.

Ferster, C. B. (1953). Sustained behavior under delayed reinforcement. Journal of Experimental Psychology, 45, 218-224.

Ferster, C. B., \& Skinner, B. F. (1957). Schedules of reinforcement. Englewood Cliffs: Prentice-Hall.

Fonseca, C. M., \& Tomanari, G. Y. (2007). Contingência e contiguidade no responder de ratos submetidos a esquemas de razão, intervalo e tempo variáveis. Interação em Psicologia, 11, 187197.

Gleeson, S., \& Lattal, K. A. (1987). Response-reinforcer relations and the maintenance of behavior. Journal of the Experimental Analysis of Behavior, 48, 383-393.

Keller, F. S., \& Schoenfeld, W. N. (1950). Principles of psychology. New York: Appleton-Century-Crofts.
Lattal, K. A. (1987). Considerations in the experimental analysis of reinforcement delay. Em M. L. Commons, J. E. Mazur, J. A. Nevin \& H. Rachlin (Orgs.), Quantitative analyses of behavior: Vol. 5. The effect of delay and of intervening events on reinforcement value (pp. 107-123). Hillsdale: Erlbaum.

Lattal, K. A., \& Gleeson, S. (1990). Response acquisition with delayed reinforcement. Journal of Experimental Psychology: Animal Behavior Process, 16, 27-39.

Lattal, K. A., \& Ziegler, D. R. (1982). Briefly delayed reinforcement: An interresponse time analysis. Journal of the Experimental Analysis of Behavior, 37, 407-416.

Morgan, M. J. (1970). Fixed-interval schedules and delay of reinforcement. Quarterly of Experimental Psychology, 22, 663673.

Pierce, C. H., Handford, P. V., \& Zimmerman, J. (1972). Effects of different delay of reinforcement procedures on variableinterval responding. Journal of the Experimental Analysis of Behavior, 18, 141-146.

Richards, R. W. (1981). A comparison of signaled and unsignaled delay of reinforcement. Journal of the Experimental Analysis of Behavior, 35, 145-152.

Schneider, S. M. (1990). The role of contiguity in free-operant unsignaled delay of positive reinforcement: A brief review. The Psychological Record, 40, 239-257.

Sizemore, O. J., \& Lattal, K. A. (1977). Dependency, temporal contiguity, and response-independent reinforcement. Journal of the Experimental Analysis of Behavior, 25, 119-125.

Sizemore, O. J., \& Lattal, K. A. (1978). Unsignaled delay of reinforcement in variable-interval schedules. Journal of the Experimental Analysis of Behavior, 30, 169-175.

Williams, B. A. (1976). The effects of unsignaled delayed reinforcement. Journal of the Experimental Analysis of Behavior, 26, 441-449.

Recebido: 24/05/2009

Última revisão: 04/11/2009

Aceite final: 24/11/2009

\section{Sobre os autores:}

Cristina Moreira Fonseca: Doutora em Psicologia Experimental pela Universidade de São Paulo. Professora da Faculdade Anhanguera/ UniA (Campus Santo André/SP).

Gerson Yukio Tomanari: Doutor em Psicologia Experimental pela Universidade de São Paulo. Professor do Instituto de Psicologia da Universidade de São Paulo.

Endereço para correspondência: Prof. Gerson Yukio Tomanari - Universidade de São Paulo - Instituto de Psicologia - Av. Prof. Mello Moraes, 1721 - 05508-030 São Paulo/SP - Endereço eletrônico: tomanari@usp.br. 